\title{
Diacronie
}

Studi di Storia Contemporanea

$\mathrm{N}^{\circ} 30,2$ | 2017

Ponti fra nazioni e continenti

\section{Benito Mussolini, Il mio diario di guerra (1915-1917)}

\section{Matteo Anastasi}

\section{(2) OpenEdition}

\section{Journals}

\section{Edizione digitale}

URL: http://journals.openedition.org/diacronie/5885

DOI: 10.4000/diacronie.5885

ISSN: 2038-0925

\section{Editore}

Association culturelle Diacronie

\section{Notizia bibliografica digitale}

Matteo Anastasi, « Benito Mussolini, Il mio diario di guerra (1915-1917)», Diacronie [Online], N 30, 2 I

2017, documento 7, Messo online il 29 juillet 2017, consultato il 23 septembre 2020. URL : http:// journals.openedition.org/diacronie/5885; DOI : https://doi.org/10.4000/diacronie.5885 


\title{
Diacronie
}

Studi di Storia Contemporanea

30, 2/2017

Ponti fra nazioni e continenti: diplomazia, immaginari e conoscenze tecniche

\section{RECENSIONE: Benito MUSSOLINI, Il mio diario di guerra (1915- 1917), Bologna, Il Mulino, 2016, 225 pp.}

\author{
A cura di Matteo ANASTASI
}

Per citare questo articolo:

ANASTASI, Matteo, «RECENSIONE: Benito MUSSOLINI, Il mio diario di guerra (1915-1917), Bologna, Il Mulino, 2016, 225 pp.», Diacronie. Studi di Storia Contemporanea : Ponti fra nazioni e continenti: diplomazia, immaginari e conoscenze tecniche, 30, 2/2017, 29/7/2017,

URL: < http://www.studistorici.com/2017/07/29/anastasi_numero_30/ >

Diacronie Studi di Storia Contemporanea $\rightarrow$ http://www.diacronie.it Rivista storica online. Uscita trimestrale.

redazione.diacronie@hotmail.it

Comitato di direzione: Naor Ben-Yehoyada - João Fábio Bertonha - Christopher Denis-Delacour - Maximiliano Fuentes Codera Anders Granås Kjøstvedt - John Paul Newman - Deborah Paci - Niccolò Pianciola - Spyridon Ploumidis - Wilko Graf Von Hardenberg

Comitato di redazione: Jacopo Bassi - Luca Bufarale - Gianluca Canè - Fausto Pietrancosta - Alessandro Salvador - Matteo Tomasoni Diritti: gli articoli di Diacronie. Studi di Storia Contemporanea sono pubblicati sotto licenza Creative Commons 3.0. Possono essere riprodotti e modificati a patto di indicare eventuali modifiche dei contenuti, di riconoscere la paternità dell'opera e di condividerla allo stesso modo. La citazione di estratti è comunque sempre autorizzata, nei limiti previsti dalla legge. 


\title{
7/ RECENSIONE: Benito MUSSOLINI, Il mio diario di guerra (1915- 1917), Bologna, Il Mulino, 2016, 225 pp.
}

\author{
A cura di Matteo ANASTASI
}

È merito di uno dei più affermati storici italiani, Mario Isnenghi, l'aver riportato alla luce, restituendogli «quell'attenzione - dovuta - che gli è stata a lungo lesinata» ${ }^{1}$, il diario di guerra redatto da Benito Mussolini nei suoi tre anni al fronte durante il primo conflitto mondiale. Isnenghi si è occupato di Grande guerra fin dai suoi primi passi nel mondo accademico, con molteplici opere legate a quel periodo ${ }^{2}$.

In questa circostanza Isnenghi ha inteso riconferire dignità storiografica all'esperienza bellica vissuta al fronte dal futuro Duce d'Italia, di sicura influenza sulla sua formazione personale e sulla sua visione politica dei decenni a seguire. Come ricorda nel testo in esame lo stesso storico veneziano, l'idea di riportare in auge i diari ha accompagnato il suo percorso di studioso per circa un trentennio, durante il quale questa convinzione non ha perso smalto, conoscendo anzi costante vigore, fino a concretizzarsi nel gennaio 2016 con la pubblicazione di questo lavoro ${ }^{3}$.

Richiamato alle armi il 31 agosto 1915 e assegnato inizialmente come soldato semplice al $7^{\circ}$ reggimento bersaglieri, Mussolini raggiunse i soldati italiani il 13 settembre, aggregandosi all'11 bersaglieri. Il 28 dicembre del 1915 «Il Popolo d'Italia» - il quotidiano da lui diretto e fondato poco più di un anno prima, il 15 novembre 1914, per dar voce all'area interventista socialista di cui era esponente principale - iniziò la pubblicazione a puntate del Giornale di guerra, una cronaca delle ostilità densa e partecipata, che accompagnò i lettori con quindici puntate stampate in quattordici mesi. Le pubblicazioni non ebbero scadenza sistematica: sei uscirono in rapida sequenza fra il 28

\footnotetext{
${ }^{1}$ MUSSOLINI, Benito, Il mio diario di guerra (1915-1917), Bologna, Il Mulino, 2016, p. 7.

${ }^{2}$ Cfr. ISNENGHI, Mario (a cura di), La prima guerra mondiale, Bologna, Zanichelli, 1972; ID., Giornali di trincea (1915-1918), Torino, Einaudi, 1977; ID. (a cura di), Operai e contadini nella Grande guerra, Bologna, Cappelli, 1982; ID., La Grande guerra, Firenze, Giunti 1993; ID., La tragedia necessaria. Da Caporetto all'otto settembre, Bologna, Il Mulino, 1999; ID., Convertirsi alla guerra. Liquidazioni, mobilitazioni e abiure nell'Italia tra il 1914 e il 1918, Milano, Donzelli, 2015.

${ }^{3}$ «Personalmente ho cominciato a fare pubblicamente i conti con questo testo in uno dei convegni animati da Gianfranco Folena a Bressanone, nell'ambito dei lavori estivi dell'Università di Padova (1984), dedicato a Le forme del Diario e poi confluito nel n. 2 dei Quaderni di retorica e poetica diretti da Folena, Padova, Liviana, 1985: Il diario di guerra di Benito Mussolini, pp. 123-130; e nella Postfazione a Il mito della Grande guerra, Bologna, Il Mulino, 1989, una terza edizione che affida l'aggiornamento di quel lavoro del 1970 all'aggiunta delle pp. 395-439». Cfr. la prefazione del curatore in MUSSOLINI, Benito, Il mio diario di guerra, cit., p. 7.
} 
dicembre 1915 e il 9 gennaio 1916; due a maggio, quattro a luglio e tre nel febbraio 1917. Il 23 febbraio 1917 Mussolini rimase seriamente ferito durante un'esercitazione, incidente che pose fine alla sua esperienza bellica così come alla sua narrazione dal fronte.

Successivamente queste memorie furono pubblicate nel febbraio 1923 da Imperia, la casa editrice di riferimento del Partito Nazionale Fascista, col titolo Il mio diario di guerra (1915-1917). Durante il Ventennio si susseguirono altre edizioni, riportanti un testo leggermente modificato rispetto a quello del 1923, pubblicate dalla Libreria del Littorio e una versione contenuta nel primo dei dodici volumi degli Scritti e discorsi di Benito Mussolini, dati alle stampe da Hoepli nel 1934 e dal Poligrafico dello Stato nel 1939. Dopo la caduta del fascismo, il diario è stato nuovamente pubblicato nel 1961 nel trentatreesimo volume dell'Opera omnia di Mussolini curata da Edoardo Susmel prima, e dal figlio Duilio poi, per La Fenice.

«Poiché le edizioni uscite durante la vita di Mussolini presentano tutte interventi o modifiche» ${ }^{4}$, Isnenghi ha deciso di riprendere fedelmente - e questo è certamente un merito ai fini dell'analisi storiografica - le puntate pubblicate negli anni di guerra su «Il Popolo d'Italia». In appendice al diario, il curatore ha inteso raccogliere una serie di materiali, utili al lettore per giungere a una valutazione più sistematica di quanto offerto dalle righe che li precedono. Nel dettaglio, si trova la seguente documentazione: alcune fotografie comparse nell'edizione Imperia del diario, con l'esclusione di quella della casa nativa di Mussolini, ritenuta inutile nel contesto preso in esame; il brano Il nostro Direttore, introduzione alla pubblicazione de «Il Popolo d'Italia» del 23 luglio 1916 intitolata Un mese sulla difensiva fra le montagne della Carnia, contenuta nell'edizione Imperia e non rinvenibile nelle successive; i due articoli relativi al ferimento e al ricovero di Mussolini, pubblicati nell'edizione Imperia ed espunti dalle successive, Al capezzale di Benito Mussolini di Sandro Giuliani del 5 marzo 1917 e Il Re visita Benito Mussolini e i suoi compagni feriti di Raffaele Garinei, pubblicato l'8 marzo 1917; il foglio matricolare di Mussolini, pubblicato da Renzo De Felice in appendice al suo Mussolini il rivoluzionario (1883-1920).

L'opera si colloca nel filone degli studi sulla prima "conversione" di Mussolini ${ }^{6}$, un Mussolini interventista, che ha rotto con l'«Avanti!» e i socialisti "ortodossi" per propagandare le ragioni della guerra sulle pagine de «Il Popolo d'Italia»: siamo ancora nella fase del Mussolini il rivoluzionario $^{7}$, secondo la periodizzazione adottata da Renzo De Felice. Si tratta di un Mussolini estremamente toccato dall'esperienza delle masse in guerra, che fa emergere una duplice

\footnotetext{
${ }^{4}$ MUSSOLINI, Benito, Il mio diario di guerra (1915-1917), cit., p. 44.

${ }^{5}$ DE FELICE, Renzo, Mussolini il rivoluzionario (1883-1920), Torino, Einaudi, 1965, pp. 665-667.

${ }^{6}$ Oltre a quelli di Renzo De Felice, confluiti nella sua monumentale biografia di Mussolini, di sicuro interesse e fondamento scientifico per questa fase della vita, sono, fra gli altri, di vario orientamento, gli studi di BOSWORTH, Richard, Mussolini, London, Arnold, 2002; GENTILE, Emilio, Le origini dell'ideologia fascista 19181925, Bari, Laterza, 1975; MACK SMITH, Denis, Mussolini, Milano, Rizzoli, 1981; MILZA, Pierre, Mussolini, Paris, Fayard, 1999; PERFETTI, Francesco, Il movimento nazionalista in Italia 1903-1914, Roma, Bonacci, 1984.

${ }^{7}$ DE FELICE, Renzo, op. cit.
} 
personalità: da un lato il disciplinato e rispettoso soldato in divisa grigioverde, dall'altro lato il leader rivoluzionario, che matura al fronte - trasponendo le sue convinzioni sulla carta stampata del giornale di cui è direttore - la necessità di cancellare l'«io» per far spazio al «noi» di un popolo inteso come soggetto unico, privo di divisioni classiste. Un tema, quest'ultimo, d'ora in avanti mai abbandonato, cui saranno molto sensibili i reduci e gli interventisti al momento della sua ascesa nel 1922, quando da semplice soldato Mussolini diverrà capo del governo nel giro di poco più di un quinquennio. Proprio nell'eliminazione di questi paradigmi di classe e delle conseguenti antitesi di ruolo, Mussolini vede l'azione benefica della guerra, della vita comunitaria in trincea, della mescolanza dei diversi dialetti che si fondono in un'unica massa grigioverde volta al medesimo obiettivo, dando vita a livelli fino ad allora inediti di nazionalizzazione.

Il tema dell'esercito/popolo al fronte è reso in passaggi di discreta godibilità attraverso la penna di Mussolini. Si pensi, in particolare, al Mussolini "antropologo", dedito allo studio delle canzoni intonate dai soldati in trincea, che preferiscono accantonare i brani patriottici in favore di «canzoni sgorgate dall'anima primitiva del popolo», versi «rozzi» ma dotati di «una fresca vena di sentimento» ${ }^{8}$ : da Daghela ben biondina a Osteria del numero uno. Nell'analisi complessiva non va trascurata, certo - e questo Isnenghi lo sottolinea a più riprese - la necessità mussoliniana di costruire attorno a sé, mediante i suoi lettori, una narrazione quasi epica, che lo elevi a leader in prima linea (nel senso fisico del termine in questo caso) per le sorti della nazione, con uno slancio nuovo, che ha superato, ma non accantonato, quello risorgimentale (la denominazione de «Il Popolo d'Italia» ricorda, e non poco, la testata mazziniana «L'Italia del Popolo») e si propone come sua necessaria evoluzione.

L'originalità dell'opera è individuabile in molteplici aspetti. Anzitutto, il merito di Isnenghi primo studioso ad attirare l'attenzione su questo testo - è di far luce su un'opera di assoluta importanza per la comprensione dell'uomo e di alcune sue caratteristiche forgiate dall'esperienza di guerra, che Mussolini porterà a lungo con sé, e che influenzeranno negli anni a venire tanto le scelte di politica estera quanto quelle di politica domestica. Un compito, quello di Isnenghi, per decenni serenamente declinato dagli storici, attenti a non commettere l'incauto approccio alle memorie di un autore la cui impronunciabilità politica ha caratterizzato lungamente il mondo scientifico del dopoguerra.

Nondimeno - altro dato meritorio - il diario fornisce un interessante quadro del fronte: spazi, luoghi, momenti di vita quotidiana, uomini, pulsioni. L'affresco è completo e minuzioso: la narrazione procede secondo il ritmo della giornata, dalla sveglia alla ritirata, fra descrizioni paesaggistiche, azioni militari, ritratti di superiori, valutazioni dei ranci, ambizioni e aspettative dei commilitoni. Fra le numerose memorie pervenuteci dalle trincee della Prima guerra

\footnotetext{
${ }^{8}$ MUSSOLINI, Benito, Il mio diario di guerra (1915-1917), cit., p. 140.
} 
mondiale9, quelle di Mussolini, soldato fra i soldati, non vanno collocate in una posizione secondaria, oltreché per le motivazioni delineate, per la peculiarità di essere scritte da un giornalista, con l'acutezza delle osservazioni e la fluidità di scrittura proprie della categoria.

${ }^{9}$ Fra le opere pubblicate recentemente, riguardo il fronte italiano, cfr. BUGANI, Flavia, Italo Stegher. Memorie di un giovane ufficiale, Udine, Gaspari, 2016; FERRARI, Pierangelo, MACULOTTI, Giancarlo, La guerra bianca di Carlo Emilio Gadda. La permanenza in Valle Camonica nelle note del Giornale di guerra e di prigionia 1915-1916, Roccafranca, Compagnia della stampa Massetti Rodella, 2016; PUCCINI, Mario, Davanti a Trieste. Esperienze di un fante sul Carso, Milano, Mursia, 2016; STUPARICH, Giani, Guerra del '15, Macerata, Quodlibet, 2015. 


\section{L'AUTORE}

Matteo ANASTASI (1989) nel 2011 si è laureato con lode in Scienze Storiche presso l'Università Europea di Roma e nel 2014, sempre con lode, in Relazioni Internazionali presso la LUISS Guido Carli. Attualmente è dottorando di ricerca presso la LUMSA e cultore della materia in Storia contemporanea presso l'Università Europea di Roma. Si occupa di storia politica e diplomatica italiana.

URL: < http://www.studistorici.com/progett/autori/\#Anastasi > 